\title{
Treatment of pulmonary coccidioidomycosis after successful hepatitis $C$ therapy in a patient with fluconazole induced hepatotoxicity
}

\author{
Zohreh Movahedi, David Wisinger, Sorin Petre, Jyotsna Ravi, Thomas Ardiles, Renee Prevette,
} Ali Al-Yaqoobi, Abdul Nadir*

Department of Medicine, Maricopa Medical Center, Phoenix, USA

Email: ${ }^{*}$ Anadir786@aol.com

Received 12 November 2011; revised 16 January 2012; accepted 25 January 2012

\begin{abstract}
A patient with hepatitis $C$ infection and cavitary pulmonary coccidioidomycosis is reported. Treatment of hepatitis $C$ was associated with resolution of fluconazole-induced hepatotoxicity. Successful treatment of hepatitis $C$ enabled the patient to tolerate increaseing doses of fluconazole. This case highlights that hepatic toxicity of fluconazole can improve after successful treatment of hepatitis $\mathrm{C}$.
\end{abstract}

Keywords: Coccidioidomycosis; Fluconazole; Hepatotoxicity

\section{INTRODUCTION}

Coccidioidomycosis is fungal infection endemic to the southwestern United States, particular Arizona. It commonly presents as a community-acquired pneumonia. Oral azole antifungal agents such as fluconazole are the standard therapy for treatment of coccidioidomycosis $[1,2]$. Azoles are metabolized by the cytochrome P-450 enzyme (CYP) system and abnormal liver function tests (LFT) have been described with azoles [3,4]. Azole-induced LFT abnormality is usually asymptomatic and reversible after the offending medication is discontinued [5-10].

Here in we report a case of a patient with hepatitis $\mathrm{C}$ virus (HCV) infection with multiple attempts to treat pulmonary coccidiodymycosis with fluconazole which antifungal treatment could not be sustained because of LFT abnormality.

\section{CASE REPORT}

A 55-year-old Caucasian female with a past medical history of hepatitis $\mathrm{C}$ and pulmonary coccidioidomycosis was referred to the liver clinic in February 2010 for eva-

${ }^{*}$ Corresponding author. luation of abnormal LFTs.

A retrospective chart review was accomplished from 1998 to identify all events related to her LFT elevation and treatment of coccidioidomycosis as well as chronic obstructive pulmonary disease (COPD). She had prison time during 1969 to 1977; 1998, 2002; 2007 and 2009. She acknowledged use of IV, nasal and inhalational drugs since age 12 . Her substance abuse included cocaine, heroin, crack, speed, methamphetamine, methadone and marijuana. She was on MMTP (methadone maintenance treatment program) between 1995 to1998. Her drug addiction led to inconsistent follow up, sometimes for several months. Nonetheless, she kept seeking medical attention whenever she became very ill. Her last drug use was in 2009.

From 2004 to 2010, she was hospitalized several times. Each time, she presented with constitutional symptoms, cough, hemoptysis and intermittent fevers. Multiple attempts to treat coccidioidomycosis with fluconazole could not be sustained because of LFT elevation. A temporal relationship was documented between the rise and fall of liver test values with initiation and withdrawal of fluconazole respectively (Figure 1). Besides fluconazole, she was treated with other medications (Figure 2). She was also treated for pulmonary mycobacterium tuberculosis (TB) with rifampicin, isoniazid, pyrazinamide and ethambutol (RIPE) in 1998 for six months and had a brief exposure to the same regimen in 2005; however, work up for TB was negative in 1975, 1988, 1996 and 2005. She tested negative for HIV in September of 2009.

Her LFTs were normal in 2000 . A $5.5 \times 5.7 \mathrm{~cm}$ left upper lobe pulmonary cavity was present on chest CT scan in December 2004. In September 2005, she had a two-day exposure to anti TB medication with rise of LFT noted. TB medications were held and LFTs trended down and TB was ruled out. Because of a high coccidioidomycosis IgG (coccidioides) titer at 1:32, she was discharged on oral fluconazole at $200 \mathrm{mg}$ daily. The coccidioides titer in December 2005 dropped to 1:2. Then 
she was lost to follow up until March 2006, when her LFTs were noted to be elevated and fluconazole was stopped and subsequently her coccidioides titer in November 2006 rose to 1:8. From Feb 2007 till September 2009 multiple attempts to treat her with fluconazole re- sulted in similar pattern of LFT abnormality and a temporal relationship between initiation of fluconazole and elevated liver enzymes was noted. After fluconazole was held liver enzymes trended down to their baseline level (Table 1).

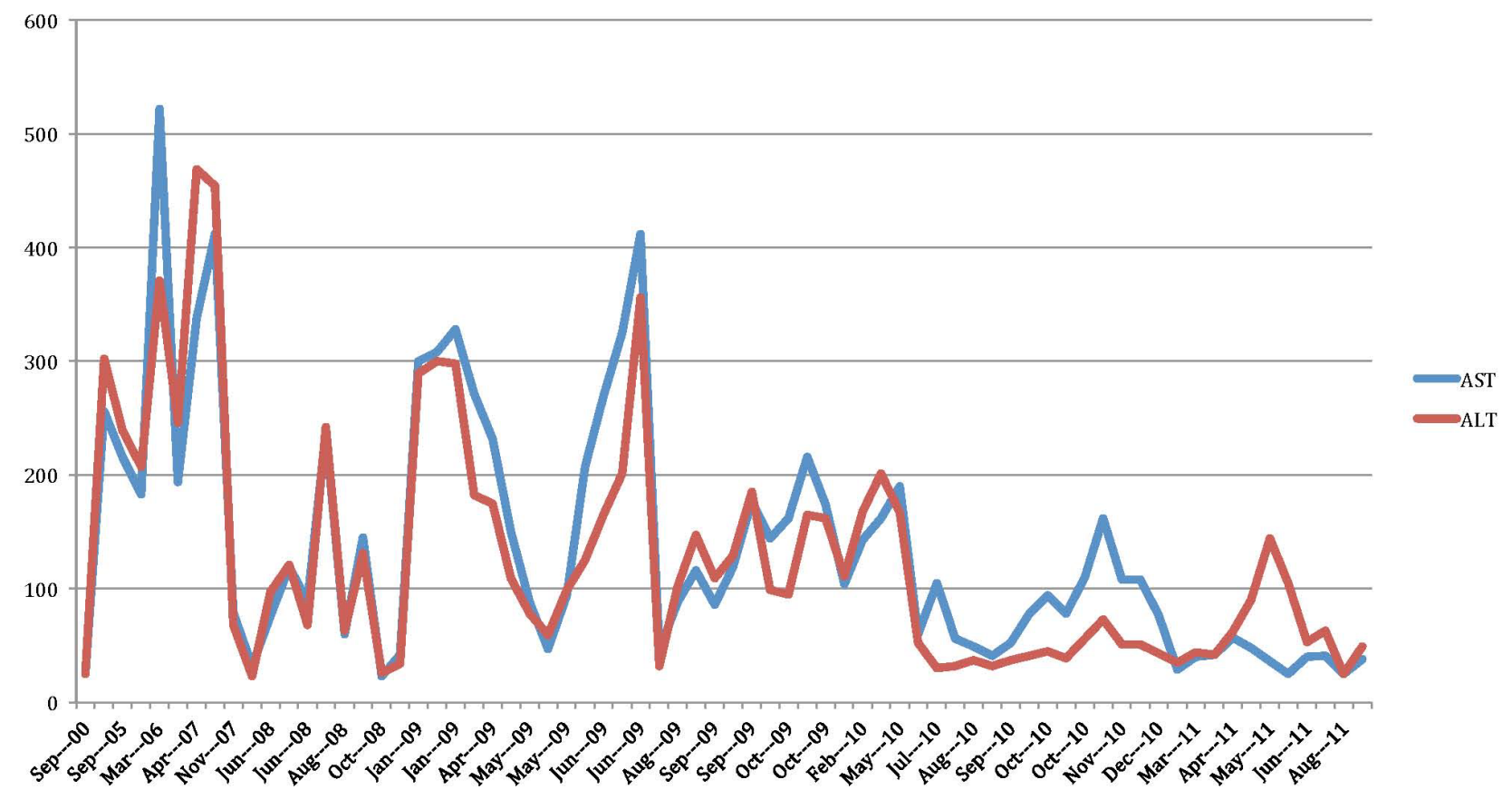

Figure 1. Liver test values over 10 years.

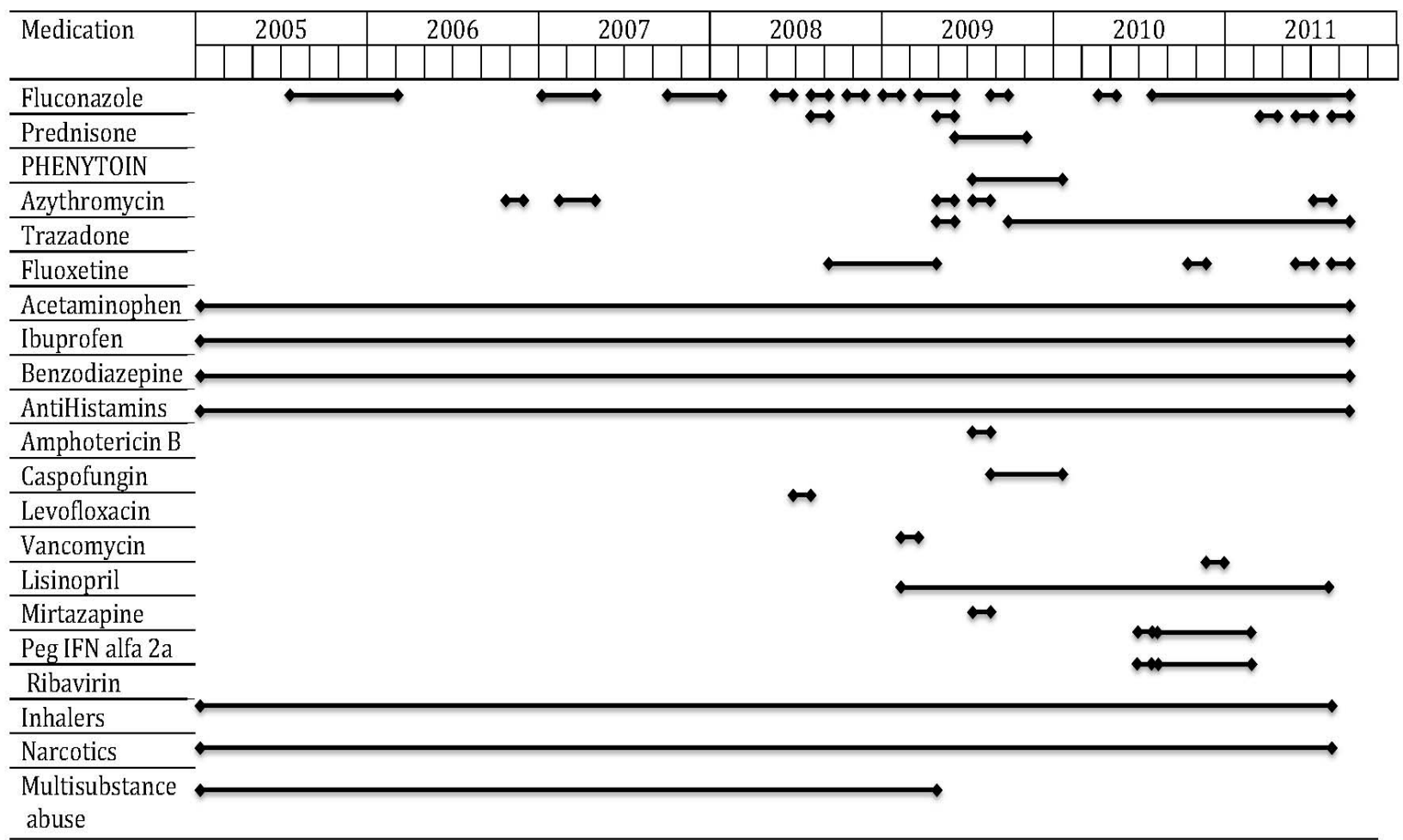

Figure 2. Medication chart showing the overlap between different medications over the course of treatment. 
Table 1. LFTs on fluconazole.

\begin{tabular}{|c|c|c|c|c|c|c|c|c|}
\hline \multicolumn{7}{|c|}{ Before HCV Treatment } & \multirow{2}{*}{$\begin{array}{c}\text { During HCV Treatment } \\
07 / 2010-12 / 2010\end{array}$} & \multirow{2}{*}{$\begin{array}{c}\text { After HCV Treatment } \\
01 / 2011-09 / 2011\end{array}$} \\
\hline Date & 2005 & 2006 & 2007 & 2008 & 2009 & 2010 & & \\
\hline \multirow{13}{*}{$\begin{array}{c}\mathrm{AST} \\
(\mathrm{U} / \mathrm{L})\end{array}$} & 256 & 522 & 338 & 32 & 300 & 143 & 60 & 29 \\
\hline & 216 & 194 & 412 & 76 & 328 & 162 & 105 & 33 \\
\hline & 183 & & 79 & 119 & 271 & 190 & 56 & 40 \\
\hline & & & & 89 & 232 & & 41 & 57 \\
\hline & & & & 238 & 150 & & 52 & 48 \\
\hline & & & & 60 & 72 & & 78 & 36 \\
\hline & & & & 145 & 74 & & 94 & 25 \\
\hline & & & & 23 & 94 & & 110 & 40 \\
\hline & & & & 42 & 207 & & 162 & 42 \\
\hline & & & & & 269 & & 85 & 44 \\
\hline & & & & & 325 & & 77 & 25 \\
\hline & & & & & 412 & & & 38 \\
\hline & & & & & 46 & & & \\
\hline \multirow{13}{*}{$\begin{array}{c}\mathrm{ALT} \\
(\mathrm{U} / \mathrm{L})\end{array}$} & 302 & 371 & 469 & 23 & 289 & 168 & 52 & 35 \\
\hline & 239 & 246 & 454 & 97 & 300 & 201 & 30 & 31 \\
\hline & 207 & & 61 & 121 & 182 & 176 & 37 & 44 \\
\hline & & & & 68 & 109 & & 27 & 62 \\
\hline & & & & 242 & 69 & & 37 & 90 \\
\hline & & & & 63 & 59 & & 45 & 144 \\
\hline & & & & 131 & 78 & & 56 & 105 \\
\hline & & & & 26 & 99 & & 39 & 64 \\
\hline & & & & 43 & 125 & & 73 & 61 \\
\hline & & & & & 165 & & 51 & 53 \\
\hline & & & & & 201 & & 43 & 25 \\
\hline & & & & & 356 & & & 49 \\
\hline & & & & & 32 & & & \\
\hline
\end{tabular}

In May 2009 coccidioides titer was noted to be at 1:64 and her left lung cavity diameter had increased to $7.8 \mathrm{~cm}$. with persistent pulmonary symptoms. A trial of amphotericin B in May 2009 for two days resulted in renal insufficiency with a creatinine rise from 0.61 to $1.67 \mathrm{mg} / \mathrm{dl}$. A wedge resection of the apical segment of left upper lobe was performed in September 2009. The resected lung specimen showed granulomatous inflammation, fungal spherules consistent with coccidiodomycosis and reactive fibrosis. Surgery was followed by a four month treatment with caspofungin.

During her clinical course, her LFTs were noted to be near normal on Aug 2008 and May 2009 when she got two, one-week courses of prednisone at doses of $10 \mathrm{mg}$ and $75 \mathrm{mg}$ daily respectively, along with fluconazole at
$200 \mathrm{mg}$ daily while she was being treated for COPD exacerbation. However, when prednisone was stopped and fluconazole reinstituted LFTs were noted to rise again in June 2009.

Upon presentation to the liver clinic on Feb 2010, she complained of productive cough, hemoptysis, fatigue, night sweats, nausea, right upper quadrant pain and shortness of breath. Her vital signs were stable, but she appeared ill. Her HCV RNA viral load was 573570 $\mathrm{IU} / \mathrm{mL}$, genotype was $3 \mathrm{a}$, and her hepatitis B Core antibody IgG was positive. The rest of her liver workup including autoimmune and metabolic liver disease was normal. A liver biopsy was performed in May 2010, and it showed early cirrhosis with mild inflammatory activity comprising of lymphocytes consistent with hepatitis C 
infection. No hepatic granulomas were seen. The complement fixation titer for coccidioidomycosis was 1:32.

In June 2010 hepatitis $\mathrm{C}$ treatment with peginterferon alpha $2 \mathrm{a}(\mathrm{IFN} \alpha)$ at $180 \mathrm{mcg}$ sub-cutaneously per week and oral ribavirin at $1200 \mathrm{mg}$ daily was initiated. Two months after starting HCV treatment, she had a significant fall of her hematocrit related to ribavirin. She was given 2 units of PRBC. Interferon and ribavirin were held for two weeks. Ribavirin was then reinstituted at $400 \mathrm{mg}$ daily, and the HCV viral load dropped to 622 IU after four weeks. Her viral load became less than 5 at 12 weeks; she completed 28 weeks of HCV treatment and achieved sustained viral response 24 weeks after stopping HCV treatment in July 2011.

The size of lung cavity had gradually increased from $5.1 \mathrm{~cm}$ diameter (post lung resection) to $7.2 \mathrm{~cm}$ diameter and her serology titer was at 1:32. Fluconazole was started again in August 2010 at a dose of $400 \mathrm{mg}$ daily along with HCV treatment. In January 2011, fluconazole was increased to $600 \mathrm{mg}$ daily, with near normal LFTs compared to the levels prior HCV treatment (Table 1). With hepatitis $\mathrm{C}$ treatment, she was able to tolerate fluconazole with favorable effect on coccidioidomycosis infection.

She showed favorable radiological, serological and clinical response; coccidioidomycosis cavity size decreased to $5.5 \mathrm{~cm}$ (Figures 3(a) and (b)), and the coccidioides titer dropped to $1: 2$.

During HCV treatment, she did not receive any prednisone tapers, however, post HCV treatment, she did receive three prednisone tapers at a dose of 40, 30 and 50 mg respectively for five, nine and five days-three, five and six months after completing HCV therapy. At the time of writing of this manuscript, she continues to receive fluconazole $600 \mathrm{mg}$ without deterioration of her liver function tests.

\section{DISCUSSION}

This patient's presentation created a clinical dilemma regarding treating her life threatening pulmonary coccidioidomycosis in the presence of underlying hepatitis $\mathrm{C}$ and cirrhosis. This case highlights that establishing drug-induced hepatotoxicity is a daunting task especially in patients who are on multiple medications with complex medical problems. Moreover, follow up is done by multiple health care providers at different places, making the correlation between elevated LFTs and the potential offending agent extremely challenging. In the described patient, her compliance was an additional caveat in documenting the effect of fluconazole on her LFTs. Nonetheless, repeated challenges with fluconazole, resulted almost invariably in elevation of LFTs, with a downward trend noted when fluconazole was held. It appears very likely that fluconazole played a significant role in the rise of her liver tests, although contribution of other agents and hepatitis $\mathrm{C}$ cannot be entirely ignored.

A recent review of azole tolerability and hepatotoxicity revealed that fluconazole has a better safety profile than other azoles and the risk of abnormal LFT requiring or not requiring treatment termination being $0.7 \%$ and $9.3 \%$ respectively [10]. In another study it was reported that patients on $800 \mathrm{mg} /$ day of fluconazole had up to $5 \%$ discontinuation because of elevated LFTs, indicating the possibility of dose dependent hepatotoxicity of fluconazole [11].

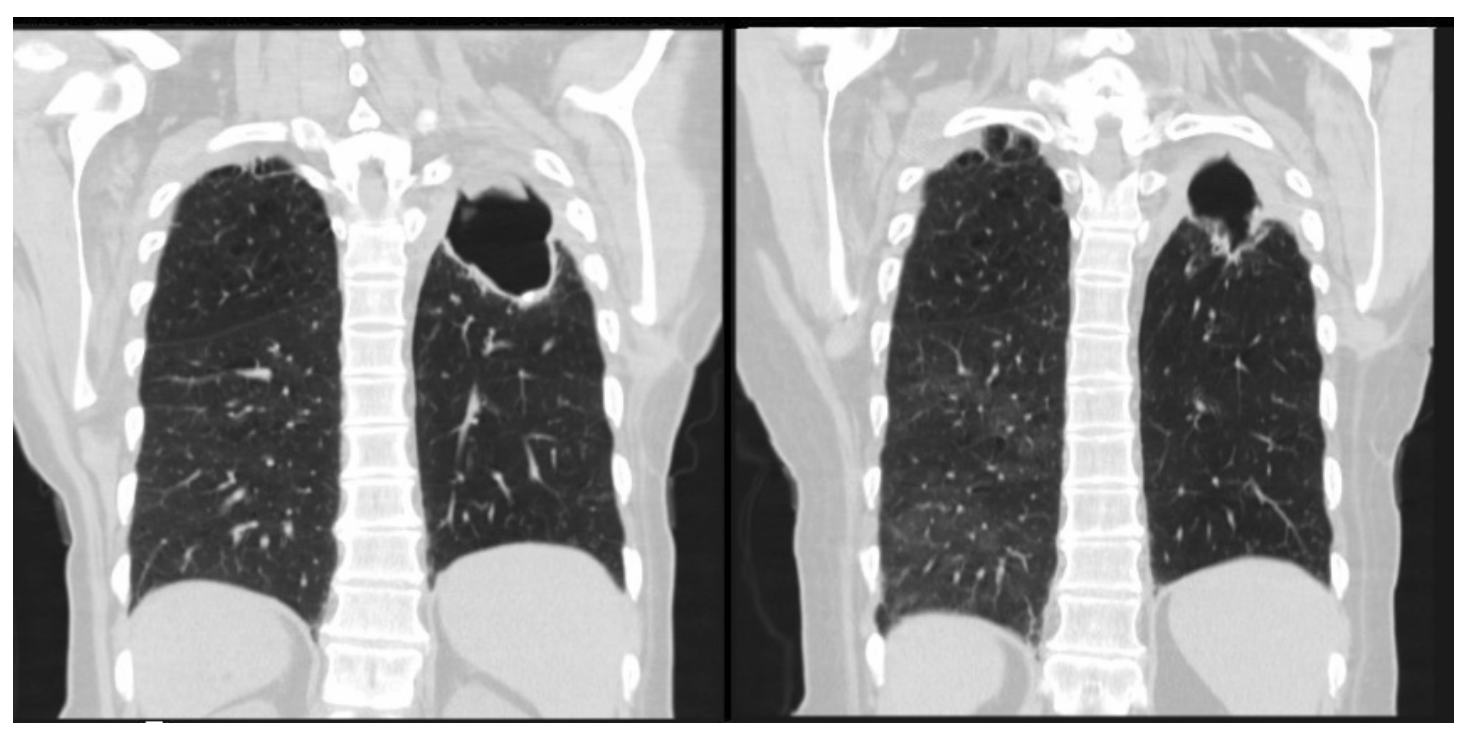

(a)

(b)

Figure 3. Axial Chest CT scan showing significant reduction in the size of the left upper lobe lung cavity. (a) After treatment; (b) Before treatment. 
Azoles disrupt the synthesis of ergosterol in fungal cell membrane through the enzyme C-14-demethylase, which is CYP dependent. Similar interactions of fluconazole and mammalian cells through enzymes dependent on CYP may mediate some of the major toxic effects of the azoles including hepatotoxicity [12]. Fluconazole inhibits CYP in a dose dependent manner. Specifically, fluconazole has been shown to be a potent inhibitor of CYP2C9 and a moderately potent inhibitor of CYP3A4, with less effect on mitochondrial function [13, 14]. On the contrary, it has been reported that pre-treating rat hepatocytes with phenobarbital (a potent inducer of cytochrome P-450) did not ameliorate fluconazole induced liver injury, thereby suggesting that the cytotoxicity of fluconazole maybe induced by mechanisms other than CYP-mediated formation of toxic metabolites [13]. Recent data also report that metabolic idiosyncrasy rather than immunologic idiosyncrasy is the key mechanism for fluconazole induced [15-18].

In HCV/HIV co-infected patients elevated LFTs have been reported. This may be the result of altered CYP content in the liver seen in patients with chronic viral hepatitis and mitochondrial toxicity experienced by $\mathrm{HCV} / \mathrm{HIV}$ co-infected patients from their ART (antiretroviral therapy). Liver fibrosis in patients with hepatitis $\mathrm{C}$ seems to be an important determinant of the risk of hepatotoxicity and successful treatment of hepatitis $\mathrm{C}$ has been reported to reduce the liver toxicity of ART [1924].

Recently, it has been shown that IFN $\alpha$ down regulates CYP3A4 expression through JAK-STAT pathway [25]. Based on this observation, one can speculate that in this case the toxicity of fluconazole was reduced during HCV treatment because of down regulation of cytochrome enzyme system. In this case after HCV was cured and treatment with IFN was discontinued, the combined inhibitory effect of HCV and IFN on the cytochrome P-450 systems was removed thus enabling the patient to tolerate higher doses of fluconazole. Once hepatitis $\mathrm{C}$ was treated, her liver tests became normal or near normal despite the fact that she was receiving up to three times the dose of fluconazole as well as many other agents she was exposed to before.

On a couple of occasions before HCV treatment and after HCV treatment, her LFTs were near normal while she was on fluconazole and prednisone. Prednisone has been shown to improve liver function tests in many conditions including autoimmune hepatitis, drug hepatitis and viral hepatitis [26]. It is certainly possible that institution of prednisone decreased inflammation of the liver and improves liver tests. However, post treatment of $\mathrm{HCV}$, she was had only a brief exposure of prednisone and even in the absence of prednisone her liver tests remained normal or near normal.
To our knowledge, this is the first reported case showing successful treatment of hepatitis $\mathrm{C}$ in a patient with cirrhosis and pulmonary coccidioidomycosis, improved fluconazole-induced hepatotoxicity significantly. This case is unique because the patient was able to tolerate fluconazole after treatment of hepatitis $\mathrm{C}$ and shows that analogous to improved hepatic tolerance of ART after successful treatment of $\mathrm{HCV} / \mathrm{HIV}$ co-infected patients hepatitis $\mathrm{C}$ treatment of patients with coccidiodomycosis can improve fluconazole induced hepatotoxicity and enable patients to take this potentially lifesaving medication.

\section{REFERENCES}

[1] Ampel, N.M. (2010) New perspectives on coccidioidomycosis. Proceedings of the American Thoracic Socirty, 7,181-185. doi:10.1513/pats.200907-080AL

[2] Ampel, N.M. (2010) Coccidioidomycosis: A review of recent advances. Clinics in Chest Medicine, 30, 241-251. doi:10.1016/j.ccm.2009.02.004

[3] Grant, S.M. and Clissold, S.P. (1990) Fluconazole: A review of its pharmacodynamic and pharmacokinetic properties, and therapeutic potential in superficial and systemic mycoses. Drugs, 39, 877-916. doi:10.2165/00003495-199039060-00006

[4] Silling, G. (2002) Fluconazole: Optimized antifungal therapy based on pharmacokinetics. Mycoses, 45, 39-41. doi:10.1111/j.1439-0507.2002.tb04768.x

[5] Song, J.C. and Deresinski S. (2005) Hepatotoxicity of antifungal agents. Current Opinion in Investigational Drugs, 6, 170-177.

[6] Gearhart, M.O. (1994) Worsening of liver function with fluconazole and review of azole antifungal hepatotoxicity. The Annuals of Pharmacotherapy, 28, 1177-1181.

[7] Wells, C. and Lever, A.M. (1992) Dose-dependent fluconazole hepatotoxicity proven on biopsy and rechallenge. Journal of Infection, 24, 111-112. doi:10.1016/0163-4453(92)91346-D

[8] Kim, H. and Bindslev-Jensen, C. (2004) Reported case of severe hepatotoxicity likely due to fluconazole and not desloratadine. Acta Haematologica, 112, 177-178. doi:10.1159/000079733

[9] Girois, S.B., Chapuis, F., Decullier, E. and Revol, B.G. (2005) Adverse effects of antifungal therapies in invasive fungal infections: Review and meta-analysis. European Journal of Clinical Microbiology \& Infectious Disease, 24, 119-130. doi:10.1007/s10096-005-1281-2

[10] Wang, J.L., Chang, C.H., Young-Xu, Y. and Chan, K.A. (2010) Systematic review and meta-analysis of the tolerability and hepatotoxicity of antifungals in empirical and definitive therapy for invasive fungal infection. Antimicrobial Agents and Chemotherapy, 54, 2409-2419. doi:10.1128/AAC.01657-09

[11] Rex, J.H., Pappas, P.G. and Karchmer, A.W. (2003) A randomized and blinded multicenter trial of high-dose 
fluconazole plus placebo versus fluconazole plus amphotericin B as therapy for candidemia and its consequences in nonneutropenic subjects. Clinical Infectious Diseases, 36, 1221-1228.

[12] Joly, V., Bolard, J. and Yeni, P. (1992) In vitro models for studying toxicity of antifungal agents. Antimicrobial Agents and Chemotherapy, 36, 1799-1804.

[13] Somchit, N., Hassim, S.M. and Samsudin, S.H. (2002) Itraconazole- and fluconazole-induced toxicity in rat hepatocytes: A comparative in vitro study. Human \& Experimental Toxicology, 21, 43-48. doi:10.1191/0960327102ht208oa

[14] Rodriguez, R.J. and Acosta Jr., D. (1995) Comparison of ketoconazole- and fluconazole-induced hepatotoxicity in a primary culture system of rat hepatocytes. Toxicology, 96, 83-92. doi:10.1016/0300-483X(94)02911-D

[15] Rodriguez, R.J. and Acosta Jr., D. (1996) Inhibition of mitochondrial function in isolated rate liver mitochondria by azole antifungals. Journal of Biochemical Toxicology, 11, 127-131. doi:10.1002/(SICI)1522-7146(1996)11:3<127::AID-JBT 4>3.0.CO;2-M

[16] Somchit, N., Ngee, C.S., Yaakob, A., Ahmad, Z. and Zakaria, Z.A. (2009) Effects of cytochrome p450 inhibitors on itraconazole and fluconazole induced cytotoxicity in hepatocytes. Journal of Toxicology, Article ID: 912320. doi: $10.1155 / 2009 / 912320$

[17] Somchit, N., Norshahida, A.R., Hasiah, A.H., Zuraini, A., Sulaiman, M.R. and Noordin, M.M. (2004) Hepatotoxicity induced by antifungal drugs itraconazole and fluconazole in rats: A comparative in vivo study. Human \& Experimental Toxicology, 23, 519-525. doi:10.1191/0960327104ht479oa

[18] Sun, G., Thai, S.F. and Lambert, G.R. (2006) Fluconazole-induced hepatic cytochrome P450 gene expression and enzymatic activities in rats and mice. Toxicology Lettes, 164, 44-53. doi:10.1016/j.toxlet.2005.11.015

[19] Guillaume, M.P., De Prez, C. and Cogan, E. (1996) Sub- acute mitochondrial liver disease in a patient with AIDS: Possible relationship to prolonged fluconazole administration. The American Journal of Gastroenterology, 91 , 165-168.

[20] Labarga, P., Soriano, V. and Vispo, M.E. (2007) Hepatotoxicity of antiretroviral drugs is reduced after successful treatment of chronic hepatitis C in HIV-infected patients. The Journal of Infectious Diseases, 196, 670-676. doi:10.1086/520092

[21] Aranzabal, L., Casado, J.L. and Moya, J. (2005) Influence of liver fibrosis on highly active antiretroviral therapy-associated hepatotoxicity in patients with HIV and hepatitis C virus coinfection. Clinical Infectious Disesses, 40, 588-593. doi:10.1086/427216

[22] Uberti-Foppa, C., De Bona, A. and Morsica, G., (2003) Pretreatment of chronic active hepatitis $\mathrm{C}$ in patients coinfected with HIV and hepatitis $\mathrm{C}$ virus reduces the hepatotoxicity associated with subsequent antiretroviral therapy. Journal of Acquired Immune Deficiency Syndromes, 33, 146-152. doi:10.1097/00126334-200306010-00005

[23] Sulkowski, M.S. and Benhamou, Y. (2007) Therapeutic issues in HIV/HCV-coinfected patients. Journal of Viral Hepatitis, 14, 371-386. doi:10.1111/j.1365-2893.2006.00816.x

[24] Sulkowski, M.S. (2004) Drug-induced liver injury associated with antiretroviral therapy that includes HIV-1 protease inhibitors. Clinical Infectious Disesses, 38, S90S97. doi:10.1086/381444

[25] Flaman, A.S., Gravel, C., Hashem, A.M., Tocchi, M. and Li, X. (2011) The effect of interferon-alpha on the expression of cytochrome P450 3A4 in human hepatoma cells. Toxicology and Applied Pharmacology, 253, 130136. doi:10.1016/j.taap.2011.03.019

[26] Tanner, A.R. and Powell, L.W. (1979) Corticosteroids in liver disease: Possible mechanisms of action, pharmacology, and rational use. Gut, 20, 1109-1124. doi:10.1136/gut.20.12.1109 